\title{
Traffic Light Switching by Traffic Density Measurement using Image Processing Technique
}

\author{
Mrs. Harshitha $\mathbf{R}^{1}$, Chandan $\mathbf{R}^{2}$, Poornima $K^{3}$, Navyashree $\mathbf{U ~ N}^{4}$, Sandesh Gowda $\mathbf{P}^{5}$ \\ Assistant Professor, ECE, GMIT, Mandya, India ${ }^{1}$ \\ $8^{\text {th }}$ Semester, ECE, GMIT, Mandya, India ${ }^{2,3,4,5}$
}

\begin{abstract}
Traffic light control is one of the serious technical hazards of the urban areas in almost every country around the world. This is due to rapid increase in number of vehicles in order to reduce the time and complexity. This newly developed project will enable the traffic light to switch from red to green based on traffic density. The simplest way for controlling a traffic light uses timer for each phase since, we proposed a system for controlling the traffic light by image processing. The system will detect vehicles through images and the image sequence will then be analysed using digital image processing for vehicle detection, and according to traffic conditions on the road traffic light can be controlled.
\end{abstract}

Keywords: Traffic light, Image Processing, edge detection.

\section{INTRODUCTION}

In India traffic is growing four times faster than population. Nowadays, many countries suffer from the traffic congestion problems that affect the transportation system in cities and cause serious dilemma. In spite of replacing traffic officers and flagmen by automatic traffic systems, the optimization of the heavy traffic jam is still a major issue to be faced, especially with multiple junction nodes.

Traffic jams also create many other critical issues and problems which directly affect the human routine lives and sometime reason for life loss for example if there is an emergency vehicle like ambulance on the road with the critical patient on board. In that situation if an ambulance gets stuck in a heavy traffic jam then there are high chances that the patient cannot reach the hospital on time. So it is very important to design an intelligent traffic system which controls traffic intelligently to avoid accidents, collisions and traffic jams.

If one lane has less traffic and the other lane with huge traffic but the duration of green light for both lanes is same then this is the waste of available resources and is inefficient. By considering the above example if the lane with higher traffic density should switch on the green signal light for a longer period than the lane with lesser density.This technique is based on the measurement of the traffic density by correlating the live traffic image with a reference image. The higher the difference is, higher traffic density is detected.

However, the traffic problem is very complicated due to the involvement of diverse parameters. First, the traffic flow depends on the time of the day where the traffic peak hours are generally in the morning and in the afternoon. On the days of the week where weekends reveal minimum load while Mondays and Fridays generally show dense traffic oriented from cities to their outskirts and in reverse direction respectively and time of the year as holidays and summer. Secondly, the current traffic light system is implemented with hard coded delays where the light transition time slots are fixed regularly and do not depend on real time traffic flow. The third point is concerned with the state of one light at an intersection that influences the flow of traffic at adjacent intersections. Also, the conventional traffic system a crucial issue is related to the smooth motion through intersections of emergency vehicles of higher priorities such as ambulances, rescue vehicles, fire brigade, police and V.I.P persons that could get stuck in the crowd.

The conventional traffic system needs to be upgraded to solve the severe traffic congestion, alleviate transportation troubles, reduce traffic volume and waiting time, minimized over all travel time and expand the benefits in health, economic and environmental sectors. This paper proposes a simple low cost, traffic light control system that aims to overcome many defects and improve that traffic management.

\section{RELATED WORK}

This system has used MATLAB, toolbox and $\mathrm{C}++$ compiler for image and video processing. In this system live video is converted into grey scale images for image processing. It also considers empty road image for comparison as a reference image. On basis of reference image, image subtraction technique is applied for extraction of traffic parameter. After that it calculates the size of a matrix and adds all values present in matrix of subtracted image. 
After adding all values of remaining matrix, it is divided by a constant. Constant is calculated by considering height of camera of road [1]. Many works predict the density of the traffic based on image processing methodology. But these techniques require the acquisition of good images whose quality are weather dependent, especially with the rain and the fog. Other researchers use sophisticated algorithms to model the various states of the traffic such as fuzzy logic [2].

The technique is proposed to control the traffic signal by using image processing, in which they first selected the reference image which is the image with no vehicles or less vehicles and every time matching real time images with that reference image. On the basis of the percentage of matching traffic lights controlled. But in this technique image matching is performed by the edge detection. The reference subtraction is a complex technique, with limited outcomes. This paper presents a density analyzer scheme based on counting the number of vehicles in the present image, which provides us more accurate information for signal decision making [3].

It involves a manual analysis of data by the traffic management team to determine the traffic light duration in each of the junction. When the time allocated for a specific path has been exhausted, the red light will be ON meaning stop and next line will be ON (green light) which means the vehicle in that path should start moving. When time is about to be exhausted, the yellow light will be $\mathrm{ON}$ in the third path informing the vehicles in that path to be ready to move, and after some second the green light will be ON. Disadvantage is traffic light timing is fixed [4].

In this technique is proposed to design an intelligent traffic system, which is based on four lane system in which time is allocated according to the number of vehicles on the lane. This paper also proposes an emergency vehicle detection, within a limited scenario [5]. In this technique is proposed which is based on neural networks, which identify the vehicles and traffic density by processing the traffic videos. The technique proposed in is based on computing the traffic load by comparing two images, the reference image and the live traffic image. They improved object detection using image segmentation and noise removal operations [6-7]. Using GPRS 3G technology, through this, we can provide a smooth flow for the ambulance by controlling the traffic light according to ambulance location to reach the hospital. The location of the ambulance can be easily identified with the help of GPS unit installed in it [8].

In addition to qualitative description of road congestion, image measurement can provide qualitative description of traffic status including speeds, vehicle counts etc... moreover, quantitative traffic parameters can give us complete traffic flow information which fulfills the requirement of traffic management theory. Image tracking of moving vehicles can give us quantitative description of traffic flow [9]. Due to traffic congestions there is also an increasing cost of transportation because of wastage of time and extra fuel consumption. Traffic jams also create many other critical issues and problems which directly affect the human routine lives and sometime reason for life loss [10].

\section{METHODOLOGY}

The design of traffic control system is based on some image processing technique.

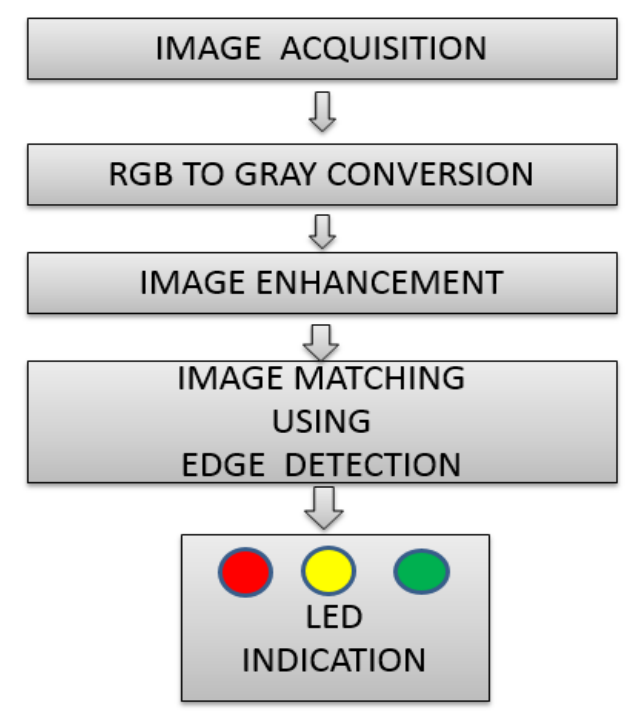

A. Image aquisition

Generally an image is a two-dimensional function $\mathrm{f}(\mathrm{x}, \mathrm{y})$ (here $\mathrm{x}$ and $\mathrm{y}$ are plane coordinates). The amplitude of image at any point say $\mathrm{f}$ is called intensity of the image. It is also called the gray level of image at that point. We need to convert these $\mathrm{x}$ and $\mathrm{y}$ values to finite discrete values to form a digital image. The input image is a taken from stare data 
base and drive data base. The image of the road with traffic is taken for processing and to check the traffic on either side of the road. Each digital image composed of a finite elements and each finite element is called a pixel. The figure is the captured by a sensor. Here photo diode sensor is used. The sensor is constructed with silicon material. The output voltage waveform of sensor is proportional to light. We can also use filter to improve selectivity. We can also make a output which has one strong color than remaining visible colors using filter. We can generate a 2-D image using single sensor with a displacement in both directions of plane. The arrangement used here is for capturing real time traffic image with the help of cameras mounted on signal poles. We can use four static camera or on single camera that will be rotated through some mechanical system. This mechanical rotation provides displacement in one direction. Images captured through these cameras will be passed to system for further processing.

\section{B. RGB to grey level}

The RGB image is first converted into grayscale image by eliminating the hue and saturation information while retaining the illumination. Hue means alter the balance of color for example: shifted to yellow instead of blue. Saturation means alter the strength of the color, when image will be de saturates the MONOCHROME image will obtain that is black and white image. Here also resize the image to the pixel range 256X256.

\section{Image enhancement}

Is the process of adjusting the digital images to more suitable for display or further image analysis, for example remove noise, sharpen or brighten an image.

The acquired image in RGB is first converted into grey. Now we want to bring our image in contrast to background so that a proper threshold level may be selected while binary conversion is carried out. This calls for image enhancement techniques. The objective of enhancement is to process the image so that result is more suitable than the original image for the specific application. There are many technique that may be used to play with the features in an image but may not be used in every case. Listed below are a few fundamental functions used frequently for image enhancement.

- Linear (negative and identity transformations)

- Logarithmic (log and inverse log transformations)

- Power law transformations (gamma correction)

- Piecewise linear transformation functions

The third method that is power law transformation has been used in this work. The power law transformations have the basic form.

$\mathbf{S}=\mathbf{c r}^{\gamma}$

Where $\mathrm{S}$ is output gray level, $\mathrm{r}$ is input gray level, $\mathrm{c}$ and $\gamma$ are positive constants. For various values of gamma applied on an acquired image we obtained the following graph shown in below figure.

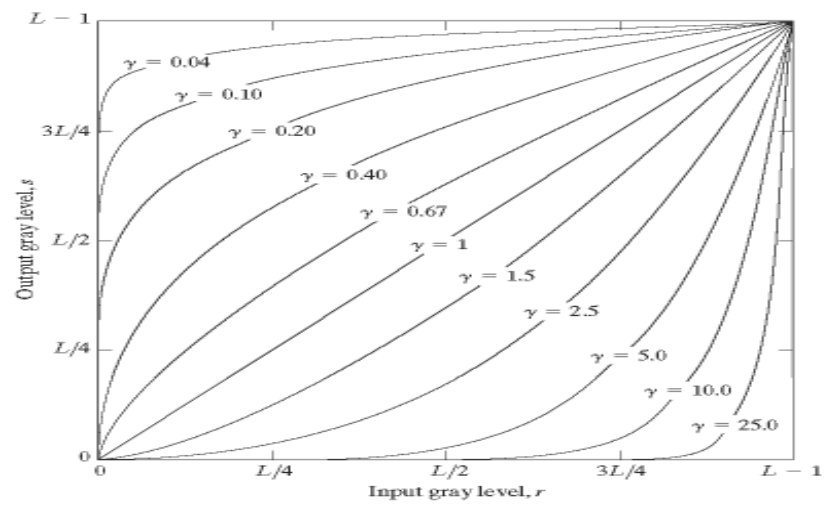

From this figure it is evident that the power law curves with fractional values of $\gamma$ map a narrow range of dark input values into a wide range of output values with the opposite being true for higher values of input levels. It depicts the effect of increasing values of $\gamma>1$. The images are shown with $\gamma=1,2,3,4,5$ as may be seen, the figure with $\gamma=1$ gives the best results in terms of making fine details identifiable. As is evident the fractional values of $\gamma$ cannot be used since these attempts show a reverse effect of brightening the image still further which we may find as undesirable for the present case.

D. Edge detection and image matching

Step 1: Edge detection: Among the key features of an image i.e. edges, lines, and points, we have used edge in our present work which can be detected from the abrupt change in the gray level. 


\section{IJIREEICE

An edge essentially demarcates between two distinctly different regions, which means that an edge is the border between two different regions.

Here we are using edge detection method for image matching:

- Edge detection methods locate the pixels in the image that correspond to the edges of the objects seen in the image.

- The result is a binary image with the detected edge pixels.

- Common algorithms used are Sobel, Prewitt and Laplacian operators.

We have used gradient based Edge Detection that detects the edges by looking for the maximum and minimum in the first derivative of the image.

- First derivative is used to detect the presence of an edge at a point in an image.

- Sign of the second derivative is used to determine whether an edge pixel lies on the dark or light side of an edge.

Gradient operators require two masks, one to obtain the $\mathrm{X}$-direction gradient and the other to obtain the $\mathrm{Y}$ direction gradient. These two gradients are combined to obtain a vector quantity whose magnitude represents the strength of the edge gradient at a point in the image and whose angle represents the gradient angle. The edge detection operator we have used in the present work is Prewitt.

Step 2: Image matching: Edge based matching is the process in which two representatives (edge) of the same objects are compared together. Any edge or its representation on one image is compared and evaluated against all the edges on the other image. Edge detection of reference and the real time images has been done using Prewitt operator. Then these edge detected images are matched and accordingly the traffic light durations can be set.

\section{E. GUI}

A graphical user interface (GUI) is a graphical display in one or more windows containing controls, called components, which enable a user to perform interactive tasks the user of the GUI does not have to create a script or type commands at the command line to accomplish the task. Unlike coding programs to accomplish task, the user of a GUI need not to understand the details of how the tasks are performed. GUI components can include menus, tool bars, pushbuttons, radio buttons, list boxes, and sliders - just to name a few. GUI is created using MATLAB tools can also perform any type of computation, read and write data files, communicate with other GUIs, and display data as tables or plots.

\section{RESULT}

Experiments are carried out and depending upon the intensity of the traffic on the road we get the following results regarding on time durations of various traffic lights.

Result 1: Matching between 10 to $50 \%$ - green light on for 60 seconds

Result 2: Matching between 50 to $70 \%$ - green light on for 30 seconds

Result 3: Matching between 70 to $90 \%$ - green light on for 20 seconds

Result 4: Matching between 90 to $100 \%$ - green light on for 10 seconds.

Result 5: Matching equal to $100 \%$ - red light on for 60 seconds.

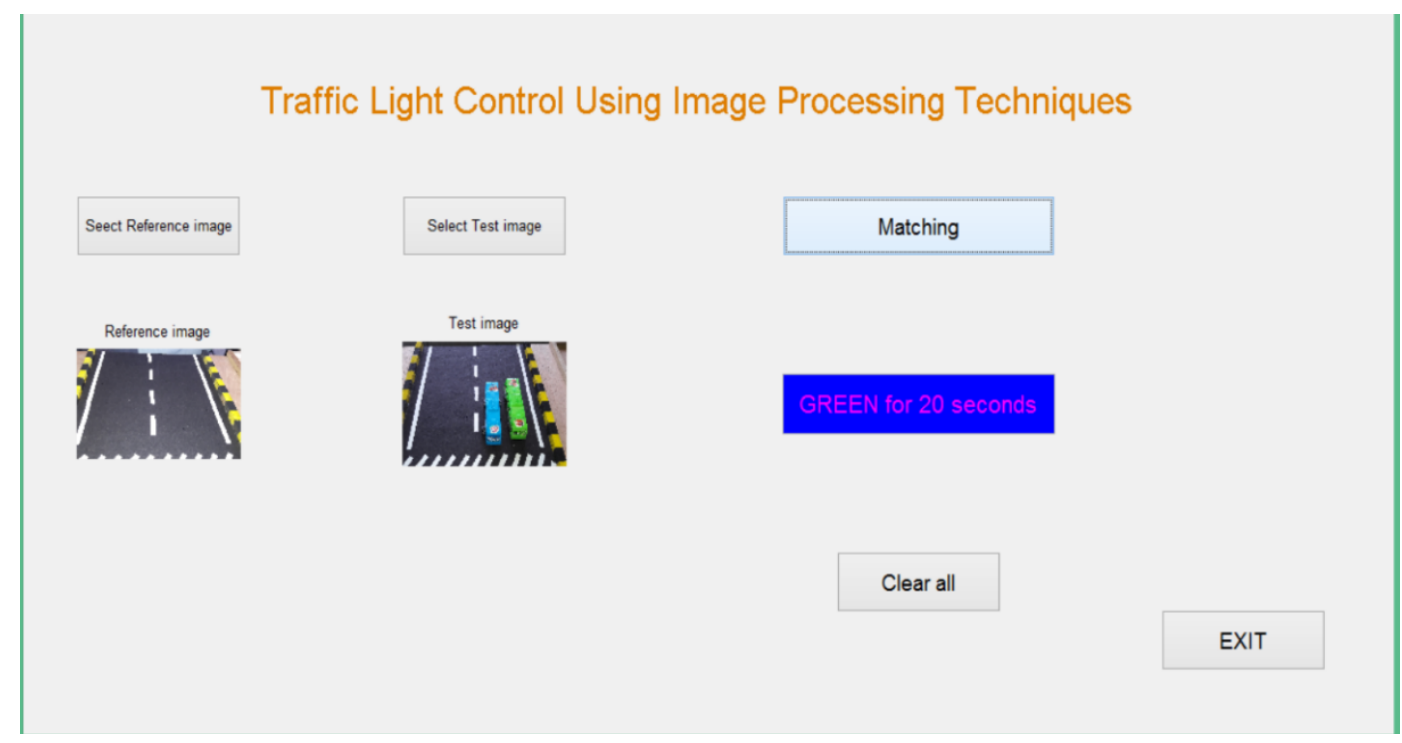




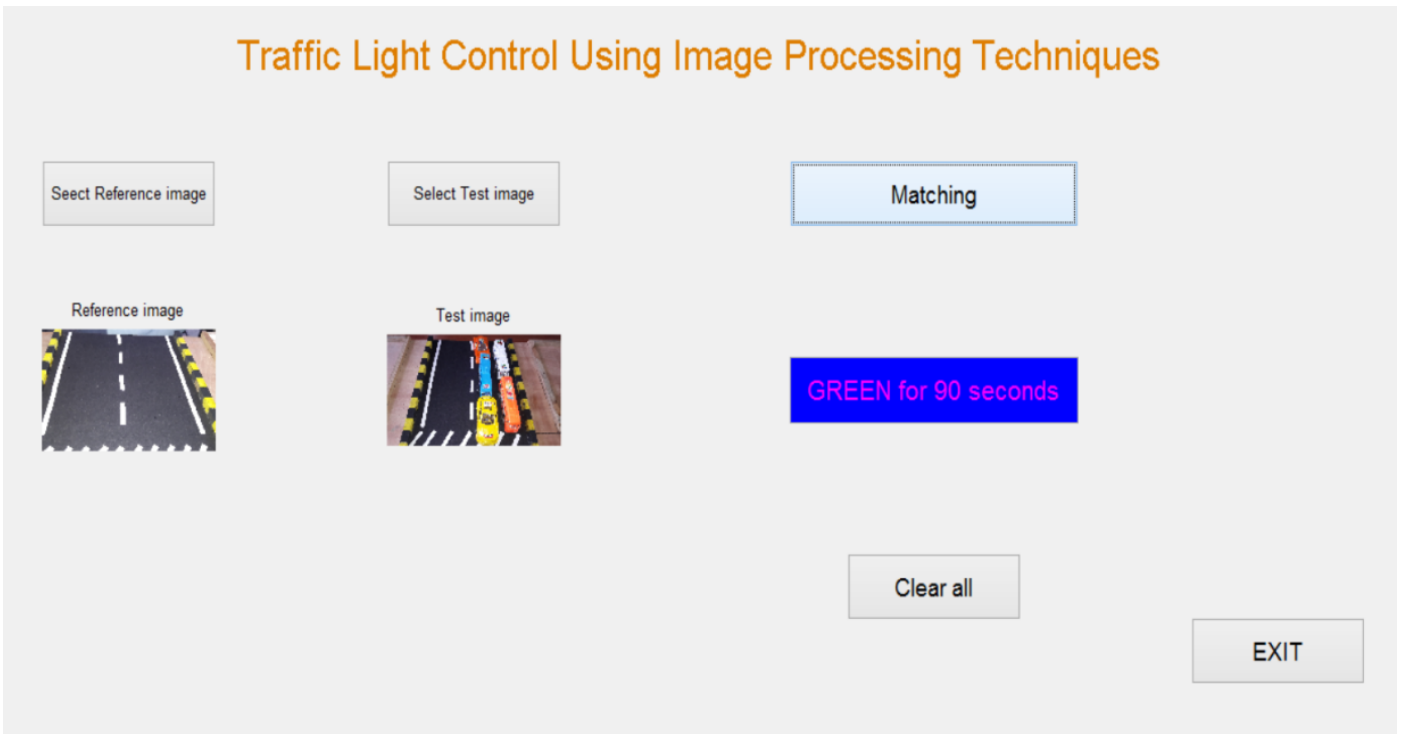

\section{CONCLUSION}

The study showed that image processing is a better technique to control the state change of the traffic light. It shows that it can reduce the traffic congestion and avoids the time being wasted by a green light on an empty road. It is also more consistent in detecting vehicle presence because it uses actual traffic images. The advantages of this proposed technique is that there is no need to use aerial imagery or complex sensor based system. The proposed system is very cost effective as it does not require installation of any additional devices, such as RFIDs. This work can be enhanced further by proposing a system which identifies the presence of emergency vehicles and by giving preference to those emergency vehicles.

\section{REFERENCES}

[1] A. Kanungo, A. Sharma and C. Singla., "Smart traffic lights switching and traffic density calculation using video processing" in IEEE International Conference on Engineering and Computational Sciences (RAECS), 2014.

[2] O.Chinyere, O. Francisca, and O.Amano, "Design and simulation of an intelligent traffic control system", International journal of advances in engineering \&technology, Vol. 1,Issue 5,pp. 47-57, Nov.2011.

[3] V. Kastrinaki, M. Zervakis, and K. Kalaitzakis, "A survey of video processing techniques for traffic applications," Indian Journal of Computer Science and Engineering (IJCSE), vol. 21, pp. 359-381, Apr 12003.

[4] Ganiyu R. A., Arulogun O.T., Okediran O.O. "Development of A Microcontroller Based Traffic Light System For Road Intersection Control" INTERNATIONAL JOURNAL OF SCIENTIFIC \& TECHNOLOGY RESEARCH VOLUME 3,ISSUE 5, MAY 2014 (2002)

[5] K. Wang, Z. Li, Q. Yao, W. Huang, and F. Wang, "An automated vehicle counting system for traffic surveillance," IEEE Int.Conf. on Vehicular Electronics and Safety, Japan, Dec 2007, pp. 1-6.

[6] Sonu Agrawal, Sushil Kumar, Sangeeta Agrawal, “A Survey on Face Recognition Techniques”, in the proceedings of International Journal of Engineering Research and Applications (IJERA - 2012), Volume 2, Issue 4, July 2012.

[7] SonamSaluja, AradhanaKumari Singh, Sonu Agrawal, "A Study of Edge-Detection Methods" International journal of advanced research in Computer and Communication Engineering, Volume 2, Issue 1, January 2013

[8] G.Sathya, Fathima Shameema S,Jyothi MolSebastian, Jemsya K S “Automatic Rescue System for Ambulance and Authoritative Vehicles, Vol 2-Issue 4 April

[9] N. J. Ferrier, S. M. Rowe, A. Blake, "Real-time traffic monitoring," Proceedings of the Second IEEE Workshop on Applications of Computer Vision, pp.81-88, 1994.

[10] Arif A. Bookseller, 2Rupali R Jagtap "Image processing based Adaptive Traffic Control System" IOSR Journal of Electronics and Communication Engineering (IOSR-JECE) ISSN: 2278-2834, ISBN: 2278-8735, 2014

\section{BIOGRAPHIES}

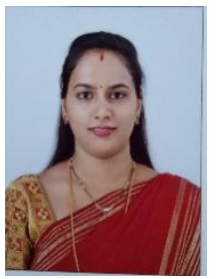

Mrs. Harshitha R Assistant Professor, Electronics and communication Dept., G Madegowda Institute of Technology Bharathinagara (K M Doddi), Mandya. 


\section{International Journal of Innovative Research in} Electrical, Electronics, Instrumentation and Control Engineering

ISO 3297:2007 Certified

Vol. 5, Issue 5, May 2017

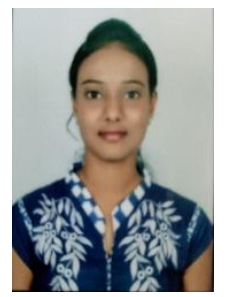

Ms. Poornima K $8^{\mathrm{TH}}$ Semester Electronics and communication Dept, G Madegowda Institute of Technology Bharathinagara (K M Doddi), Mandya.

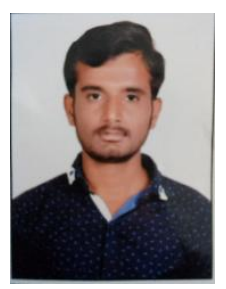

Mr. Chandan $\mathbf{R} 8^{\mathrm{TH}}$ Semester Electronics and communication Dept. G Madegowda Institute of Technology, Bharathinagara (K M Doddi), Mandya.

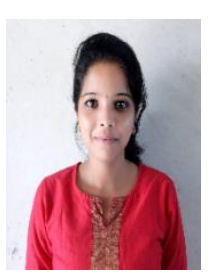

Ms. Navyashree U N $8{ }^{\mathrm{TH}}$ Semester Electronics and communication Dept. G Madegowda Institute of Technology Bharathinagara (K M Doddi), Mandya.

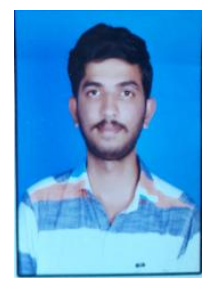

Mr. Sandesh gowda P $8^{\mathrm{TH}}$ Semester Electronics and communication Dept. G Madegowda Institute of Technology, Bharathinagara (K M Doddi), Mandya. 